\title{
CANCER
}

\section{Herbal medicine reduces chemotherapy toxicity}

The toxic side effects of anticancer drugs can be a major limitation to their effective use, as well as seriously affecting quality of life. Now, Lam and colleagues report the beneficial effects of a herbal mixture known as PHY906 in reducing the gastrointestinal toxicity associated with the DNA topoisomerase inhibitor irinotecan, which is a second-line treatment for colon and rectal carcinomas.

Diarrhoea and nausea are common side effects of irinotecan, and ulceration and intestinal bleeding can occur following its administration as the drug causes cell death and inflammation in the intestine. However, current approaches to manage these toxicities have limited effectiveness.

PHY906, which is derived from an ancient Chinese medicine used to treat nausea, vomiting and diarrhoea, has been shown to reduce diarrhoea and nausea in initial trials in patients receiving irinotecan. It consists of a mixture of four herbs, quality controlled for consistency.

To investigate how PHY906 relieves irinotecan-induced intestinal damage, the authors used a mouse model of colorectal cancer. Tumour-bearing mice were treated with irinotecan in combination with PHY906 for 4 days. PHY906 treatment alone did not inhibit tumour growth but it potentiated the antitumour activity of irinotecan and decreased the weight loss associated with its use. In addition, PHY906 promoted the recovery and regeneration of intestinal cells that were damaged by irinotecan.
Further investigations showed that although PHY906 did not prevent irinotecan-induced DNA damage in intestinal cells, it reduced the number of apoptotic cells and increased the number of proliferative cells in the intestine following irinotecan treatment. PHY906 also promoted the expression of intestinal progenitor or stem cell markers, such as CD44, leucine-rich repeatcontaining $\mathrm{G}$ protein-coupled receptor 5 and ASCL2, following irinotecan treatment.

Stimulation of the Wnt signalling pathway may be implicated in the observed regeneration of intestinal cells, as PHY906 increased the expression of key Wnt constituents, including WNT3, frizzled 5 and lowdensity lipoprotein receptor-related protein 5, following irinotecan administration. Studies in human embryonic kidney 293 cells also showed increased WNT3A activity following PHY906 treatment.

Finally, the authors showed that PHY906 inhibited several steps of irinotecan-provoked inflammation. PHY906 treatment blocked the infiltration of neutrophils and macrophages and reduced the expression of pro-inflammatory cytokines, such as tumour necrosis factor- $\alpha$, in the intestine. Particular elements of PHY906 were also discovered to potently inhibit nuclear factor- $\kappa \mathrm{B}$ and its downstream mediators cyclooxygenase 2 and inducible nitric oxide synthase.

So, although PHY906 does not provide protection from chemotherapy-induced gastrointestinal damage, it does stimulate

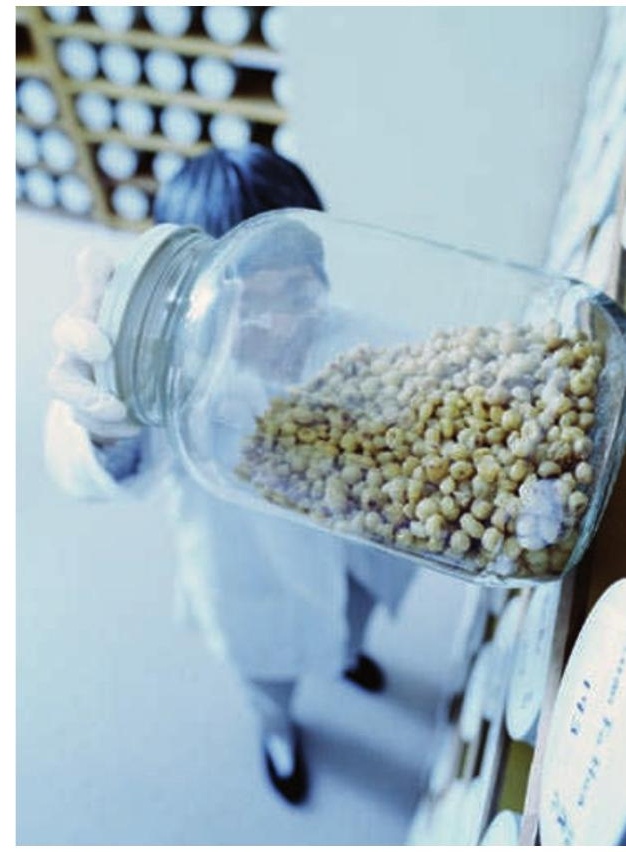

the recovery of intestinal cells by promoting progenitor cell repopulation and inhibiting inflammatory responses. This study highlights the potential value of traditional herbal medicines for which consistent manufacturing protocols can be established, and illustrates the likely mechanistic basis of such products - the modulation of multiple biological activities by multiple components of the medicines - which might provide therapeutic effects that are not currently achievable with conventional drugs.

Kate Hedigan

ORIGINAL RESEARCH PAPER Lam, W. et al. The four-herb Chinese medicine PHY906 reduces chemotherapy-induced gastrointestinal toxicity. Sci. Transl. Med. 2, 45ra59 (2010) 